\title{
Study on Fish Diversity of Kawardha Town, Chhattisgarh, India
}

\author{
Sachin Sahu ${ }^{1^{*}}$ and Subhendu Datta ${ }^{2}$ \\ ${ }^{I}$ Director of Fisheries, District- Kabirdham, Chhattisgarh, India \\ ${ }^{2}$ ICAR-Central Institute of Fisheries Education, Kolkata Center, India \\ *Corresponding author
}

\section{A B S T R A C T}

\begin{tabular}{|l|}
\hline Ke y w o r d s \\
$\begin{array}{l}\text { Fish diversity, } \\
\text { Sakari river, } \\
\text { Freshwater, } \\
\text { Kawardha }\end{array}$ \\
\hline Article Info \\
\hline $\begin{array}{l}\text { Accepted: } \\
\text { 20 August } 2020 \\
\text { Available Online: } \\
\text { 10 September } 2020\end{array}$ \\
\hline
\end{tabular}

\section{Introduction}

Kawardha is the historical and peaceful town, have wide diversity of fish. Fish are the half of total vertebrate among the biodiversity which contributes the GDP. It promotes foreign exchange earnings, income generation, food and nutritional security of the nation. Regional investigation of fish diversity is essential for documentation as well as for conservation. It will help farmers and local community to aware about their resources. Om Prakash (2004), Singh (2004), Dev (2008) has done riverine biodiversity, Choubey and Qureshi (2013) had worked on particular town Rajnandgaon. Patel et al.,
(2016) worked from different water resources in Raigarh district. The present study was undertaken to know the fish diversity of Kawardha town of Chhattisgarh.

\section{Materials and Methods}

A total four lentic (Ponds; Bade Talab, BhojaliTalab, KaliTalab, KhalheTalab) and one lotic (Sakari river) water resources were selected. The study was carried out for seven months (September2019 to March 2020). The samples were collected from Sakaririver near Saigona village and Paliguda village, landing centers, local fishermen and selected ponds from cooperative societies of Kawardha 
Town. The collected species were identified on the field itself and the unidentified specimen brought to laboratory and were preserved in $6 \%$ formalin and identified with the help of books and keys (Day. 1878, Datta and Shrivastava, 1988, Talwar and Jhingran, 1991). Water qualities were measured by the standard methods (APHA, 1998).

\section{Results and Discussion}

Water quality parameters were found suitable during the study period for fish culture. During the study period, total of 54 species belonging to 20 families under seven orders and 39 genera were recorded. Out of 54 species recorded (Table 1), three species $(5.5 \%)$ had ornamental value and 19 species
(35.18\%) have both ornamental and food value (Fig. 2). Family Cyprinidae dominated among the groups of ornamental and food fish category with eight species followed by Channidae three species, Ambassidae two species and others eight families with one species from each group (Fig. 1). Sahu (2015) had found 100 ornamental fish species in river Mahanadi. Mahapatra and Lakra (2014) had recorded 41 indigenous ornamental fishes from east Kolkata wetlands. Choubey and Qureshi (2013) has worked on ichthyofunal biodiversity of Rajnandgaon town and found total 45 species and majority was from family Cyprinidae. Patel et al., (2016) reported total 61 species under 41 genera, 22 families and seven orders from Raigarh district.

Table.1 Fish diversity survey of Kabirdham district, CG and their Commercial importance

\begin{tabular}{|c|c|c|c|c|c|}
\hline Family & Scientific Name & $\begin{array}{l}\text { Common } \\
\text { Name }\end{array}$ & Local name & $\begin{array}{l}\text { Conservat } \\
\text { ion status }\end{array}$ & $\begin{array}{l}\text { Commercia } \\
\text { importance }\end{array}$ \\
\hline \multicolumn{6}{|c|}{ 1) Order- Cypriniformes } \\
\hline \multirow[t]{17}{*}{ Cyprinidae } & $\begin{array}{l}\text { Amblypharyngodon mola (Hamilton, } \\
\text { 1822) }\end{array}$ & Molacarplet & Mohroli & $\mathrm{LC}$ & FF/OR \\
\hline & Barilius bendelisis (Hamilton,1807) & $\begin{array}{l}\text { Hamilton } \\
\text { barila }\end{array}$ & Jori & $\mathrm{LC}$ & $\mathrm{FF}$ \\
\hline & Catla catla (Hamilton, 1822) & Catla & Katla & $\mathrm{LC}$ & FF \\
\hline & Cirrhinus mrigala (Hamilton, 1822) & Mrigal & Mirgal & $\mathrm{LC}$ & FF \\
\hline & Cirrhinus reba (Hamilton, 1822) & Reba carp & LohiMirgal & $\mathrm{LC}$ & $\mathrm{FF}$ \\
\hline & $\begin{array}{l}\text { Ctenopharyngodon idella } \\
\text { (Valenciennes, 1844) }\end{array}$ & Grass carp & Ghaskat & $\mathrm{NE}$ & FF \\
\hline & Cyprinus carpio (Linnaeus, 1758 & Common carp & Komalkar & VU & FF \\
\hline & Danio rerio (Hamilton, 1822) & Zebra fish & Dadai & $\mathrm{LC}$ & FF/OR \\
\hline & Garra mullya (Sykes, 1839) & Sucker fish & Gadela & LC & FF \\
\hline & $\begin{array}{l}\text { Hypophthalmichthys molitrix } \\
\text { (Valenciennes, 1844) }\end{array}$ & Silver carp & Silver Kar & NT & FF \\
\hline & $\begin{array}{l}\text { Hypophthalmichthys nobilis } \\
\text { (Richardson, 1845) }\end{array}$ & Bighead carp & Bigrad & DD & FF \\
\hline & Labeo calbasu (Hamilton, 1822) & $\begin{array}{l}\text { Orange-fin } \\
\text { labeo }\end{array}$ & Kalbaz & LC & FF/OR \\
\hline & Labeo gonius (Hamilton, 1822) & KuriaLabeo & Roha & LC & FF \\
\hline & Labeo rohita (Hamilton, 1822) & Rohu & Roha & LC & $\mathrm{FF}$ \\
\hline & Ostreobrama cotio (Hamilton,1822) & Cotio & Kotri & LC & FF/OR \\
\hline & Systomus sarana (Hamilton, 1822) & Olive barb & Kotra & $\mathrm{LC}$ & FF/OR \\
\hline & Puntius sophore (Hamilton, 1822) & Pool barb & Kotri & LC & OR \\
\hline
\end{tabular}




\begin{tabular}{|c|c|c|c|c|c|}
\hline & Pethia ticto( Hamilton, 1822) & Ticto barb & Kotri & $\mathrm{LC}$ & OR \\
\hline & Salmophasia bacaila (Hamilton,1822) & $\begin{array}{l}\text { Large razor } \\
\text { belly } \\
\text { minnow }\end{array}$ & Sarangi & LC & $\mathrm{FF}$ \\
\hline & $\begin{array}{l}\text { Epalzeorhynchos bicolor } \\
\text { (Smith,1931) }\end{array}$ & $\begin{array}{l}\text { Red-tailed } \\
\text { black shark }\end{array}$ & --- & EN & OR \\
\hline Balitoridae & Acanthocobitis botia (Hamilton,1822) & $\begin{array}{l}\text { Mottled } \\
\text { Loach }\end{array}$ & Rudwa & $\mathrm{LC}$ & FF \\
\hline Cobitidae & $\begin{array}{l}\text { Lepidocephalichthys guntea } \\
\text { (Hamilton,1822) }\end{array}$ & Guntea loach & Rudai & $\mathrm{LC}$ & OR/FF \\
\hline \multicolumn{6}{|c|}{ 2) Order- Siluriformes } \\
\hline \multirow[t]{3}{*}{ Siluridae } & Ompokbima culatus (Bloch, 1794) & Butter catfish & Pabda & NT & FF/OR \\
\hline & Ompok pabda (Hamilton, 1822) & Pabdah cat fish & Pabda & NT & FF \\
\hline & $\begin{array}{l}\text { Walla goattu } \text { (Bloch \& } \\
\text { Schneider,1801) }\end{array}$ & Wallago & Padhina & NT & FF \\
\hline Sisoridae & Gagata gagata (Hamilton, 1822) & GengeticGagata & -.- & $\mathrm{LC}$ & $\mathrm{FF}$ \\
\hline Schilbeidae & $\begin{array}{l}\text { Eutropiichthys murius (Hamilton, } \\
\text { 1822) }\end{array}$ & Butchua & Golmuhi & $\mathrm{LC}$ & $\mathrm{FF}$ \\
\hline \multirow[t]{5}{*}{ Bagridae } & Sperata aor (Hamilton, 1822) & $\begin{array}{l}\text { Long- } \\
\text { whiskeredcatfish }\end{array}$ & $\begin{array}{ll}\text { h } & \text { Tengana } \\
\end{array}$ & $\mathrm{LC}$ & FF \\
\hline & Mystus cavasius (Hamilton, 1822) & Gangeticmystus & DesiTengna & $\mathrm{LC}$ & FF \\
\hline & Speratase enghala (Sykes, 1839) & $\begin{array}{l}\text { Giant river- } \\
\text { catfish }\end{array}$ & Tengna & $\mathrm{LC}$ & FF \\
\hline & Mystus tengara (Hamilton, 1822) & Tengara catfish & Tengna & LC & FF \\
\hline & Neotropius atherinoides (Bloch,1794) & Indian potasi & $\begin{array}{l}\text { JaliyaTenga } \\
\text { na }\end{array}$ & $\mathrm{LC}$ & FF/OR \\
\hline \multirow[t]{2}{*}{ Pangasiidae } & $\begin{array}{l}\text { Pangasius pangasius } \\
\text { (Hamilton,1822) }\end{array}$ & Pangas catfish & Sawali & $\mathrm{LC}$ & FF \\
\hline & Ailia coila (Hamilton, 1822) & Gangeticailia & Dadwa & NT & FF \\
\hline $\begin{array}{l}\text { Heteropneusti } \\
\text { dae }\end{array}$ & Heteropneustes fossilis (Bloch, 1794) & Stinging catfish & Singhi & LC & FF/OR \\
\hline \multirow[t]{2}{*}{ Clariidae } & Clarias batrachus(Linnaeus, 1758) & Walking catfish & Mongri & $\mathrm{LC}$ & FF/OR \\
\hline & Clarias gariepinus(Burchell,1822) & $\begin{array}{l}\text { North Africa } \\
\text { Catfish }\end{array}$ & Mangur & $\mathrm{LC}$ & FF \\
\hline \multicolumn{6}{|c|}{ 3) order- Clupeiformes } \\
\hline Clupeidae & Gudusia chapra (Hamilton, 1822) & $\begin{array}{l}\text { Indian river } \\
\text { shed }\end{array}$ & Chandaini & $\mathrm{LC}$ & FF \\
\hline Engraulididae & Gonialos amanmina (Hamilton, 1822) & $\begin{array}{l}\text { Ganges river } \\
\text { gizzard } \\
\text { shad }\end{array}$ & Chandaini & LC & FF \\
\hline \multicolumn{6}{|c|}{ 4) order- Osteoglossiformes } \\
\hline \multirow[t]{2}{*}{ Notopteridae } & Notopterus notopterus (Pallas,1769) & $\begin{array}{l}\text { Bronze } \\
\text { featherback }\end{array}$ & Chital & LC & FF/OR \\
\hline & Notopterus chitala (Hamilton, 1822) & $\begin{array}{l}\text { Indian } \\
\text { featherback }\end{array}$ & Chital & NT & FF/OR \\
\hline \multicolumn{6}{|c|}{ 5) Order- Anabantiformes } \\
\hline Channidae & Channa gachua (Hamilton, 1822) & $\begin{array}{l}\text { Dwarf } \\
\text { snakehead }\end{array}$ & Bhunda & LC & FF \\
\hline
\end{tabular}




\begin{tabular}{|c|c|c|c|c|c|}
\hline & Channa marulius (Hamilton, 1822) & $\begin{array}{l}\text { Great } \\
\text { snakehead }\end{array}$ & Bhunda & LC & FF/OR \\
\hline & Channa punctate (Bloch, 1793) & $\begin{array}{l}\text { Spotted } \\
\text { snakehead }\end{array}$ & Bhunda & LC & FF/OR \\
\hline & Channas triata(Bloch, 1793) & $\begin{array}{l}\text { Striped } \\
\text { snakehead }\end{array}$ & $\begin{array}{l}\text { Bhunda/ } \\
\text { Demchul }\end{array}$ & $\mathrm{LC}$ & FF/OR \\
\hline \multicolumn{6}{|c|}{ 6)Order- Perciformes } \\
\hline \multirow[t]{2}{*}{ Ambassidae } & Chanda nama (Hamilton, 1822 & $\begin{array}{l}\text { Ganges river } \\
\text { sprat }\end{array}$ & Chandaini & $\mathrm{LC}$ & FF \\
\hline & Chanda ranga (Hamilton,1822) & $\begin{array}{l}\text { Elongate } \\
\text { glass-perchlet }\end{array}$ & Chandaini & $\mathrm{LC}$ & $\mathrm{FF} / \mathrm{OR}$ \\
\hline Nandidae & Nandus nandus (Hamilton, 1822) & $\begin{array}{l}\text { Gangetic } \\
\text { leaffish }\end{array}$ & Talafia & $\mathrm{LC}$ & $\mathrm{FF}$ \\
\hline \multirow[t]{2}{*}{ Cichlidae } & $\begin{array}{l}\text { Oreochromis mossambicus } \\
\text { (Peters,1852) }\end{array}$ & $\begin{array}{l}\text { Mozambique } \\
\text { tilapia }\end{array}$ & Talafia & NT & $\mathrm{FF} / \mathrm{OR}$ \\
\hline & Oreochromisniloticus(Linnaeus, 1758) & Nile Tilapia & Talafia & VU & FF/OR \\
\hline Anabantidae & Anabas testudineus (Bloch, 1792) & $\begin{array}{l}\text { Climbing } \\
\text { perch }\end{array}$ & Kevai & $\mathrm{DD}$ & $\mathrm{FF}$ \\
\hline Belonidae & Xenentodon cancila (Hamilton,1822) & $\begin{array}{l}\text { Freshwater } \\
\text { garfish }\end{array}$ & Bama & $\mathrm{LC}$ & $\mathrm{FF}$ \\
\hline \multicolumn{6}{|c|}{ 7) Order- Synbranchiformes } \\
\hline \multirow[t]{2}{*}{$\begin{array}{l}\text { Mastacembeli } \\
\text { dae }\end{array}$} & $\begin{array}{l}\text { Mastacembelus armatus (Lacepède, } \\
1800 \text { ) }\end{array}$ & Zig-zag eel & Bami & $\mathrm{LC}$ & FF \\
\hline & $\begin{array}{l}\text { Macrognathus pancalus (Hamilton, } \\
1822\end{array}$ & $\begin{array}{l}\text { Barred } \\
\text { spiny eel }\end{array}$ & Bami & LC & $\mathrm{FF} / \mathrm{OR}$ \\
\hline
\end{tabular}

DD = Data Deficient, EN= Endangered, LC = Least Concern, NE= Not Evaluated, NT = Near Threatened, VU= Vulnerable, $\mathrm{FF}=$ Foodfish, $\mathrm{OR}=$ Ornamental fish

Fig.1 Family wise fish species in number

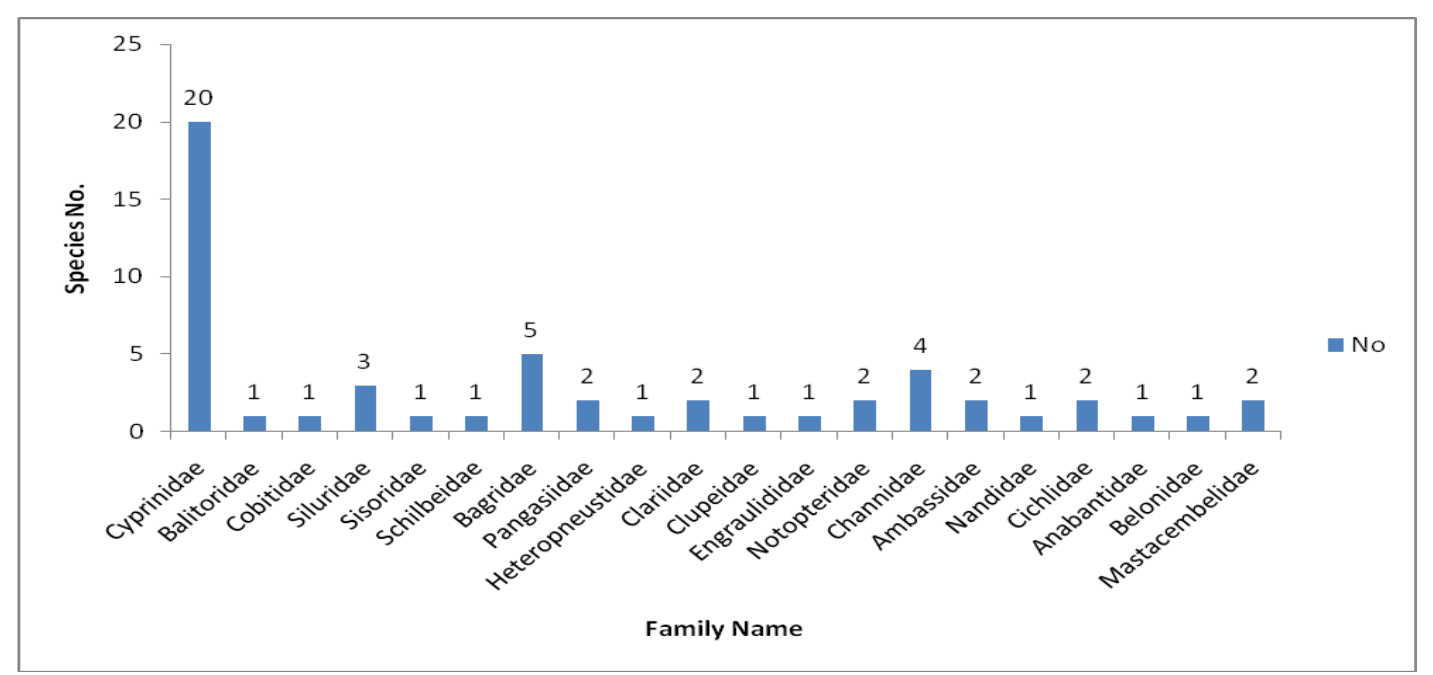


Fig.2 Percentage composition of ornamental and food species

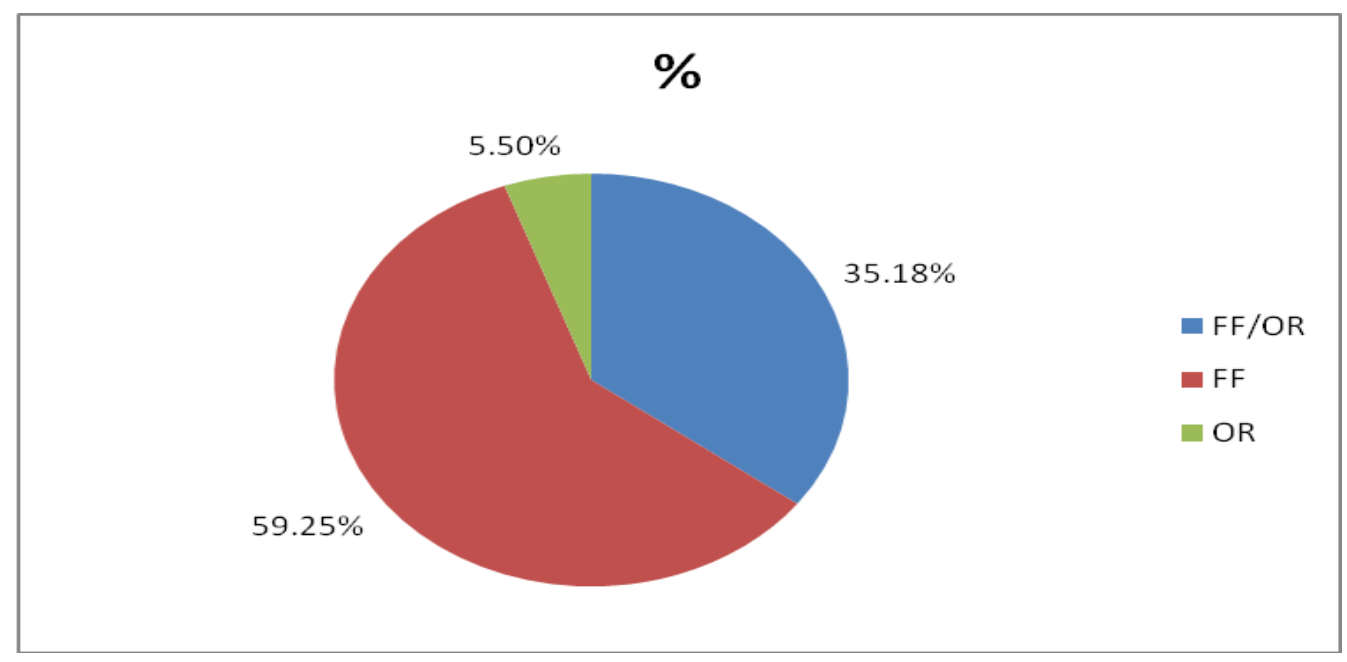

The present study found that wide variety of fish species in Kawardha town of Chhattisgarh.

In conclusion the finding of the present study shows that Kawardha town has hub of multifarious fish species which have economic importance in local as well as oversees market. The fisherman unable to identify the fishes and their value, so, the local fishermen need to aware about it by organizing training or counseling which will help to get more profit and it will help to conservation.

\section{References}

APHA (1998).Standard methods for examination of water and waste water, $20^{\text {th }}$ Ed. American Public Health Association, AWWA, WPCA, Washington, D.C., U.S.A.1193p.

Choubey, K. and Qureshi, Y. (2013) Study of Ichthyofaunal Biodiversity of Rajnandgaon town, CG, India, 2(2): 2124.

Dev, R. (2008). Fish Biodiversity in the riverine resources of Durg districts of Chhattisgarh India. M.F.Sc. Thesis. Deptt. of Fisheries, IGKV, Raipur.
Mahapatra, B.K. and Lakra, W.S. (2014) Ornamental fishes of East Kolkata Westland, West Bengal, India. Zoology, 3(12): 406-408.

Om Prakash (2004).Fish diversity in the water resources of Northern part of Raipur district of Chhattisgarh state. M.F.Sc. Thesis. Deptt. of Fisheries, IGKV, Raipur.

Patel, G., Chari, M.S., Kumar, S. Bhakta, D. and Behera, S. (2016). Status of ornamental fish diversity of Raigarh district, Chhattisgarh, India, International Journal of Science and Nature, 17 (3): 575-578.

Sahu, K.R. (2015) Studies on Piscean Diversity of Mahanadi River Chandrapur, District Janjgir-Champa, Chhattisgarh. International Journal of Modern Sciences and Engineering Technology, 2(7): 1-5.

Singh, S. (2004). Fish diversity in the water resources of Southern part of Raipur district of Chhattisgarh state. M.F.Sc. Thesis. Dept. of Fisheries, IGKV, Raipur.

Talwar, P. K. and Jhingran, A.G. (1991). Inland fishes of India and adjacent countries, 2 vols. Oxford and IBH Publishing Co. Pvt. Ltd., New Delhi. $1158 \mathrm{p}$. 


\section{How to cite this article:}

Sachin Sahu and Subhendu Datta. 2020. Study on Fish Diversity of Kawardha Town, Chhattisgarh, India. Int.J.Curr.Microbiol.App.Sci. 9(09): 2949-2952. doi: https://doi.org/10.20546/ijcmas.2020.909.363 